\title{
The Application of Coupled Three Order Cumulants' Differential Feature in Fault Diagnosis
}

\author{
Wenbing Wu, Jinquan Xiong, Yupeng Wu, Rihua Liu \\ Department of Mathmatics and Computer Science, Nanchang Normal University, Nanchang, China \\ Email: wwbysq@fjnu.edu.cn
}

How to cite this paper: Wu, W.B., Xiong, J.Q., Wu, Y.P. and Liu, R.H. (2018) The Application of Coupled Three Order $\mathrm{Cu}-$ mulants' Differential Feature in Fault Diagnosis. International Journal of Modern Nonlinear Theory and Application, 7, 77-87. https://doi.org/10.4236/ijmnta.2018.72006

Received: April 29, 2018

Accepted: June 26, 2018

Published: June 29, 2018

Copyright $\odot 2018$ by authors and Scientific Research Publishing Inc. This work is licensed under the Creative Commons Attribution International License (CC BY 4.0).

http://creativecommons.org/licenses/by/4.0/

\section{(c) (i) Open Access}

\begin{abstract}
Complex three-order cumulant has different definition forms. Different forms conclude different information. For studying the effection of frequency in the coupled signals to fault diagnosis, the differential method to the three order cumulants of coupled signals is adopted. By using the differential of complex three order cumulants before and after respectively, then their $1 \frac{1}{2}$ dimensional spectrum is calculated, and the results are used to fault diagnosis. The experimental results show that, the increase frequency item in three order cumulants after differentiated impacts on the results of fault diagnosis and the degree of effection is relative to the differential times. And the correct rate of fault diagnosis can be raised by changing the differential times of three order cumulants.
\end{abstract}

\section{Keywords}

Three Order Cumulants, Differential, Coupled, $1 \frac{1}{2}$ Dimensional Spectrum, Fault Diagnosis

\section{Introduction}

The High-order statistics method which has very good inhibition for various noises is gradually becoming a new hot spot in signal processing. It is not only nonsensitive to additive noise of auto-correlation, and also is nonsensitive to non-Gauss colored noise. So it plays an important role in non-Gaussian, non-linear, non-minimum phase, non-stationary, Gaussian colored noise processing.

At present, the study of High-order cumulants has been very popular in mechanical fault diagnosis. For instance, Shao Ren-ping and others have applied 
bispectrum to gear damage detection [1] [2] [3], and Huang Yijian and others have applied trispectrum to fault diagnosis of speed regulating valve [4] [5], which has obtained better effects.

The applications of high-order spectrum above are in the range of real number signal, W. R. Raghuveer and Chinese scholars such as Wang Shuxun have made an intensive study of high order cumulants of complex signal in the amount of coupling properties, and explicitly pointed out the different definition forms of coupling characteristics of all kinds of complex high order cumulantes [6] [7] [8]. The literatures [9] and [10] use the coupling properties for the fault diagnosis of hydraulic valve. On this basis, the paper adopts the method of differential of three-order complex cumulant, in order to change the proportion of the frequency information in the amplitude of the coupled three-order cumulant.

Because $1 \frac{1}{2}$ dimension spectrum can well response signals' coupling, if $1 \frac{1}{2}$ dimension spectrum differentiated is applied to the fault diagnosis, the performance of three-order cumulant before or after it is differentiated can be studied. The experiments are carried out on many types of fault, and most results improve with the increase of differential times in a certain degree.

\section{Three Order Cumulant and Its Differential Properties}

\subsection{Real Three Order Cumulant and Its Differential Properties}

Let $\{x(n)\}$ be a real, discrete, zero-mean and k-order stationary random process, its three order cumulant can be defined as:

$$
c_{3 x}\left(\tau_{1}, \tau_{2}\right)=E\left\{x(n) x\left(n+\tau_{1}\right) x\left(n+\tau_{2}\right)\right\}
$$

where $\tau_{1}=\tau_{2}=\tau$, then its $1 \frac{1}{2}$ dimensional spectrum is defined as the Fourier transform, i.e.:

$$
B(\omega)=\sum_{\tau=-\infty}^{\infty} c_{3 x}(\tau, \tau) e^{-j \omega \tau}
$$

Let $x(n)$ in expression (1) be coupling signal, i.e.: $x(n)=\sum_{i=1}^{3} A_{i} \cos \left(\omega_{i} n+\phi_{i}\right)$, where $\phi_{3}=\phi_{2}+\phi_{1}$ and $\omega_{3}=\omega_{1}+\omega_{2}$, so $X(n)$ is a coupling signal, its three-order cumulant is

$$
\begin{aligned}
c_{3 x}\left(\tau_{1}, \tau_{2}\right)= & E\left\{x(n) x\left(n+\tau_{1}\right) x\left(n+\tau_{2}\right)\right\} \\
= & \frac{A_{1} A_{2} A_{3}}{4} \cdot\left[\cos \left(\omega_{1} \tau_{2}+\omega_{2} \tau_{1}\right)+\cos \left(\omega_{3} \tau_{1}-\omega_{1} \tau_{2}\right)\right. \\
& +\cos \left(\omega_{1} \tau_{1}+\omega_{2} \tau_{2}\right)+\cos \left(\omega_{3} \tau_{1}-\omega_{2} \tau_{2}\right) \\
& \left.+\cos \left(\omega_{1} \tau_{1}-\omega_{3} \tau_{2}\right)+\cos \left(\omega_{2} \tau_{1}-\omega_{3} \tau_{2}\right)\right]
\end{aligned}
$$

where its diagonal three-order cumulant is:

$$
c_{3 x}(\tau, \tau)=\frac{A_{1} A_{2} A_{3}}{2} \cdot\left[\cos \left(\omega_{1} \tau\right)+\cos \left(\omega_{2} \tau\right)+\cos \left(\omega_{3} \tau\right)\right]
$$


For $x(n)$ Differential after:

$$
\mathrm{d} x(n) / \mathrm{d} n=\sum_{i=1}^{3} A_{i} \omega_{i} \cos \left(\omega_{i} n+\phi_{i}+\frac{\pi}{2}\right)
$$

where its three-order cumulant becomes:

$$
\begin{aligned}
c_{3 x}\left(\tau_{1}, \tau_{2}\right)= & \frac{A_{1} A_{2} A_{3} \omega_{1} \omega_{2} \omega_{3}}{4} \cdot\left[\cos \left(\omega_{1} \tau_{2}+\omega_{2} \tau_{1}+\frac{\pi}{2}\right)+\cos \left(\omega_{3} \tau_{1}-\omega_{1} \tau_{2}+\frac{\pi}{2}\right)\right. \\
& +\cos \left(\omega_{1} \tau_{1}+\omega_{2} \tau_{2}+\frac{\pi}{2}\right)+\cos \left(\omega_{3} \tau_{1}-\omega_{2} \tau_{2}+\frac{\pi}{2}\right) \\
& \left.+\cos \left(\omega_{1} \tau_{1}-\omega_{3} \tau_{2}+\frac{\pi}{2}\right)+\cos \left(\omega_{2} \tau_{1}-\omega_{3} \tau_{2}+\frac{\pi}{2}\right)\right]
\end{aligned}
$$

For $x(n) k$ time's differential after its three-order cumulant is:

$$
\begin{aligned}
c_{3 x}\left(\tau_{1}, \tau_{2}\right)= & \frac{A_{1} A_{2} A_{3} \omega_{1}^{k} \omega_{2}^{k} \omega_{3}^{k}}{4} \cdot\left[\cos \left(\omega_{1} \tau_{2}+\omega_{2} \tau_{1}+\frac{k \pi}{2}\right)+\cos \left(\omega_{3} \tau_{1}-\omega_{1} \tau_{2}+\frac{k \pi}{2}\right)\right. \\
& +\cos \left(\omega_{1} \tau_{1}+\omega_{2} \tau_{2}+\frac{k \pi}{2}\right)+\cos \left(\omega_{3} \tau_{1}-\omega_{2} \tau_{2}+\frac{k \pi}{2}\right) \\
& \left.+\cos \left(\omega_{1} \tau_{1}-\omega_{3} \tau_{2}+\frac{k \pi}{2}\right)+\cos \left(\omega_{2} \tau_{1}-\omega_{3} \tau_{2}+\frac{k \pi}{2}\right)\right]
\end{aligned}
$$

its diagonal three-order cumulant is:

$$
c_{3 x}(\tau, \tau)=\frac{A_{1} A_{2} A_{3} \omega_{1}^{k} \omega_{2}^{k} \omega_{3}^{k}}{4} \cdot\left[\cos \left(\omega_{1} \tau+\frac{k \pi}{2}\right)+\cos \left(\omega_{2} \tau+\frac{k \pi}{2}\right)+\cos \left(\omega_{3} \tau+\frac{k \pi}{2}\right)\right]
$$

Because of the three order cumulant of formula (3) can be considered as a function of $\tau$, so that it can directly be differentiated by $\tau$, the result is:

$$
c_{3 x}(\tau, \tau)=\frac{A_{1} A_{2} A_{3}}{2} \cdot\left[\omega_{1} \cos \left(\omega_{1} \tau+\frac{\pi}{2}\right)+\omega_{2} \cos \left(\omega_{2} \tau+\frac{\pi}{2}\right)+\omega_{3} \cos \left(\omega_{3} \tau+\frac{\pi}{2}\right)\right]
$$

after $k$ times differentiation, its diagonal three-order cumulant is:

$$
c_{3 x}(\tau, \tau)=\frac{A_{1} A_{2} A_{3}}{2} \cdot\left[\omega_{1}^{k} \cos \left(\omega_{1} \tau+\frac{k \pi}{2}\right)+\omega_{2}^{k} \cos \left(\omega_{2} \tau+\frac{k \pi}{2}\right)+\omega_{3}^{k} \cos \left(\omega_{3} \tau+\frac{k \pi}{2}\right)\right]
$$

\subsection{Complex Number Three-Order Cumulant and Differential Properties}

In Equation (1), assume that $x(n)$ is a Complex signal, which is

$$
x(n)=\sum_{i=1}^{3} A_{i} \exp \left(j\left(\omega_{i} n+\phi_{i}\right)\right),
$$

where $\phi_{3}=\phi_{2}+\phi_{1}$ and $\omega_{3}=\omega_{1}+\omega_{2}$, so $X(n)$ is a coupling signal. According to literature (7), $x(n)$ takes its conjugate or not, its third-order cumulant will have different definitions, different definitions will contain different types of coupling information, seeing Table 1. In Table 1, whatever the definition, amplitude of third order cumulant does not contain frequency information, taking into account the important role of frequency information in fault diagnosis, let 
$y(n)=\mathrm{d} x(n) / \mathrm{d} n$, then in Table 2, cumulant of $y(n)$ is shown in Table 1 , let $\tau_{1}=\tau_{2}=\tau$, three-order cumulant diagonal slice $c_{3 x}(\tau, \tau)$ can be got, and $1 \frac{1}{2}$ dimension spectrum is defined as follows:

$$
B(\omega)=\sum_{\tau=-\infty}^{\infty} c_{3 x}(\tau, \tau) \mathrm{e}^{-j \omega \tau}
$$

As can be seen from the comparison of Table 1 and Table 2, after first time differential, the amplitude of three order cumulant changes from $A_{1} A_{2} A_{3}$ into $A_{1} A_{2} A_{3} \omega_{1} \omega_{2} \omega_{3}$ in two different modes of definition. Through the same analysis, when differentiated two times, the amplitude of $y(n)$ 's three-order cumulant becomes $A_{1} A_{2} A_{3} \omega_{1}^{2} \omega_{2}^{2} \omega_{3}^{2}$. So, let $z(n)=\mathrm{d} x^{k}(n) / \mathrm{d} n^{k}$, the amplitude of $z(n)^{\prime} s$ three-order cumulant becomes $A_{1} A_{2} A_{3} \omega_{1}^{k} \omega_{2}^{k} \omega_{3}^{k}$ in Table 3. In summary, with the increase of differential times, the effect of frequencies in coupling signals to the amplitude of third-order cumulant becomes greater, and the effect is the

Table 1. Three order cumulant of $\mathrm{x}(\mathrm{n})$.

\begin{tabular}{cccc}
\hline $\begin{array}{c}\text { Definition } \\
\text { mode }\end{array}$ & Definition of three-order cumulant & Three-order cumulant & Diagonal of three-order cumulant \\
\hline Definition 1 & $E\left\{x(n) x\left(n+\tau_{1}\right) x\left(n+\tau_{2}\right)\right\}$ & 0 & 0 \\
Definition 2 & $E\left\{x^{*}(n) x\left(n+\tau_{1}\right) x\left(n+\tau_{2}\right)\right\}$ & $A_{1} A_{2} A_{3}\left[\exp j\left(\omega_{1} \tau_{1}+\omega_{2} \tau_{2}\right)+\exp j\left(\omega_{2} \tau_{1}+\omega_{1} \tau_{2}\right)\right]$ & $2 A_{1} A_{2} A_{3}\left[\exp j\left(\omega_{3} \tau\right)\right]$ \\
Definition 3 & $E\left\{x^{*}(n) x\left(n+\tau_{1}\right) x^{*}\left(n+\tau_{2}\right)\right\}$ & $A_{1} A_{2} A_{3}\left[\exp j\left(\omega_{3} \tau_{1}-\omega_{1} \tau_{2}\right)+\exp j\left(\omega_{3} \tau_{1}-\omega_{2} \tau_{2}\right)\right]$ & $A_{1} A_{2} A_{3}\left[\exp j\left(\omega_{2} \tau\right)+\exp j\left(\omega_{1} \tau\right)\right]$ \\
\hline
\end{tabular}

Table 2. Three-order cumulant of $y(n)$.

\begin{tabular}{|c|c|c|c|}
\hline $\begin{array}{l}\text { Definition } \\
\text { mode }\end{array}$ & Definition of three-order cumulant & Three-order cumulant & Diagonal of three-order cumulant \\
\hline Definition 1 & $E\left\{y(n) y\left(n+\tau_{1}\right) y\left(n+\tau_{2}\right)\right\}$ & 0 & 0 \\
\hline Definition 2 & $E\left\{y^{*}(n) y\left(n+\tau_{1}\right) y\left(n+\tau_{2}\right)\right\}$ & $\begin{array}{l}A_{1} A_{2} A_{3} \omega_{1} \omega_{2} \omega_{3}\left[\exp j\left(\omega_{1} \tau_{1}+\omega_{2} \tau_{2}+\pi / 2\right)\right. \\
\left.+\exp j\left(\omega_{2} \tau_{1}+\omega_{1} \tau_{2}+\pi / 2\right)\right]\end{array}$ & $2 A_{1} A_{2} A_{3} \omega_{1} \omega_{2} \omega_{3}\left[\exp j\left(\omega_{3} \tau+\pi / 2\right)\right]$ \\
\hline Definition 3 & $E\left\{y^{*}(n) y\left(n+\tau_{1}\right) y^{*}\left(n+\tau_{2}\right)\right\}$ & $\begin{array}{l}A_{1} A_{2} A_{3} \omega_{1} \omega_{2} \omega_{3}\left[\exp j\left(\omega_{3} \tau_{1}-\omega_{1} \tau_{2}-\pi / 2\right)\right. \\
\left.+\exp j\left(\omega_{3} \tau_{1}-\omega_{2} \tau_{2}-\pi / 2\right)\right]\end{array}$ & $\begin{array}{l}A_{1} A_{2} A_{3} \omega_{1} \omega_{2} \omega_{3}\left[\exp j\left(\omega_{2} \tau-\pi / 2\right)\right. \\
\left.+\exp j\left(\omega_{1} \tau-\pi / 2\right)\right]\end{array}$ \\
\hline
\end{tabular}

Table 3. Three order cumulant of $\mathrm{z}(\mathrm{n})$.

\begin{tabular}{|c|c|c|c|}
\hline $\begin{array}{l}\text { Definition } \\
\text { mode }\end{array}$ & Definition of three-order cumulant & Three-order cumulant & Diagonal of three-order cumulant \\
\hline Definition 1 & $E\left\{z(n) z\left(n+\tau_{1}\right) z\left(n+\tau_{2}\right)\right\}$ & 0 & 0 \\
\hline Definition 2 & $E\left\{z^{*}(n) z\left(n+\tau_{1}\right) z\left(n+\tau_{2}\right)\right\}$ & $\begin{array}{l}A_{1} A_{2} A_{3} \omega_{1}^{k} \omega_{2}^{k} \omega_{3}^{k}\left[\exp j\left(\omega_{1} \tau_{1}+\omega_{2} \tau_{2}+k \pi / 2\right)\right. \\
\left.+\exp j\left(\omega_{2} \tau_{1}+\omega_{1} \tau_{2}+k \pi / 2\right)\right]\end{array}$ & $2 A_{1} A_{2} A_{3} \omega_{1}^{k} \omega_{2}^{k} \omega_{3}^{k}\left[\exp j\left(\omega_{3} \tau+k \pi / 2\right)\right.$ \\
\hline Definition 3 & $E\left\{z^{*}(n) z\left(n+\tau_{1}\right) z^{*}\left(n+\tau_{2}\right)\right\}$ & $\begin{array}{l}A_{1} A_{2} A_{3} \omega_{1}^{k} \omega_{2}^{k} \omega_{3}^{k}\left[\exp j\left(\omega_{3} \tau_{1}-\omega_{1} \tau_{2}-k \pi / 2\right)\right. \\
\left.+\exp j\left(\omega_{3} \tau_{1}-\omega_{2} \tau_{2}-k \pi / 2\right)\right]\end{array}$ & $\begin{array}{l}A_{1} A_{2} A_{3} \omega_{1}^{k} \omega_{2}^{k} \omega_{3}^{k}\left[\exp j\left(\omega_{2} \tau-k \pi / 2\right)\right. \\
\left.+\exp j\left(\omega_{1} \tau-k \pi / 2\right)\right]\end{array}$ \\
\hline
\end{tabular}


same in all above three definition ways. So do the affection to $1 \frac{1}{2}$ dimension, shown in Tables 1-3.

Through the above analysis, whatever the signal is either real or complex signal, the affect of the frequencies in coupling signals amplifies with the increases of diffenential times in fault diagnosis, and this paper will make use of this characteristic in speed control valve's fault diagnosis.

\section{Volume Dimension}

According to document [11], the box cover algorithm is used to calculate the capacity dimensions of $1 \frac{1}{2}$ dimension spectrum. The method to calculate the similarity ratio is to let the object to be measured filled with boxes, the number of boxes needed to fulfill the object is counted, then it is used to calculate the object's capacity dimension, the box size decreases from 0.1 to 0.002 . If a ruler with a length of $\mathrm{r}$ is used to measure a line segment with a length of $L$, the ratio of $L$ to $r$ is $N$. For an object with Dc dimension:

$$
N(r) \propto\left(\frac{1}{r}\right)^{D_{c}} \rightarrow \lim N(r)=\left(\frac{1}{r}\right)^{D_{c}}
$$

After taking logarithm, capacity dimension is obtained:

$$
D_{c}=\lim \left(\frac{\log N(r)}{\log \left(\frac{1}{r}\right)}\right)
$$

\section{Data Acquisition}

The mechanical vibration component studied in the experiment is speed control valve. Vibration signals are collected from this valve in normal state and different fault states successively. In the experiment, 5 kinds of faults are set up artifically. In every state, datum in each state collected depending on working oil pressure (1 - 5 $\mathrm{MPa}$ ) is divided into 5 groups. 5 kinds of artifical faults are as follows:

Fault one: spring in back of throttle valve deformed;

Fault two: spring added with foreign objects;

Fault three: cylinder iron core pulled out in back of throttle valve;

Fault four: cylinder iron core replaced with a gasket;

Fault five: combined fault two and fault four.

The number of each group of datum used in the experiment is 1024 (Figure 1).

\section{Spectrum}

In order to identify fault, according to formula (2), the 21 groups of experiment datum in normal state and fault state are used. All of their $1 \frac{1}{2}$ dimension spectrum are drawn, and three of them are shown in Figure 2, and the Volume dimensions of these $1 \frac{1}{2}$ dimension spectrum are calculated, as shown in Table 4 . 
In Figure 2, $\mathrm{X}$ axis is circular frequency and the unit is $\pi$, the vertical axis represents the magnitude of normalized $1 \frac{1}{2}$ dimensional spectrum, dimensionless.

Then the $1 \frac{1}{2}$ dimensional spectrum of the same above-mentioned three groups of datum when differentiated 1, 4 and 8 times are shown in Figures 3-5. Because the $1 \frac{1}{2}$ dimension spectrum affected by the frequency components, as shown above, with the increase of the differential times, spectrum peak of each data become more and more concentrated, more and more acute. This should be the result of frequency components affecting in three order cumulants with the increase of differential times, the $1 \frac{1}{2}$ dimension spectrum peak distribution of location, density and strength have become more and more obviously distinctive between two groups of datum in normal state and fault state.

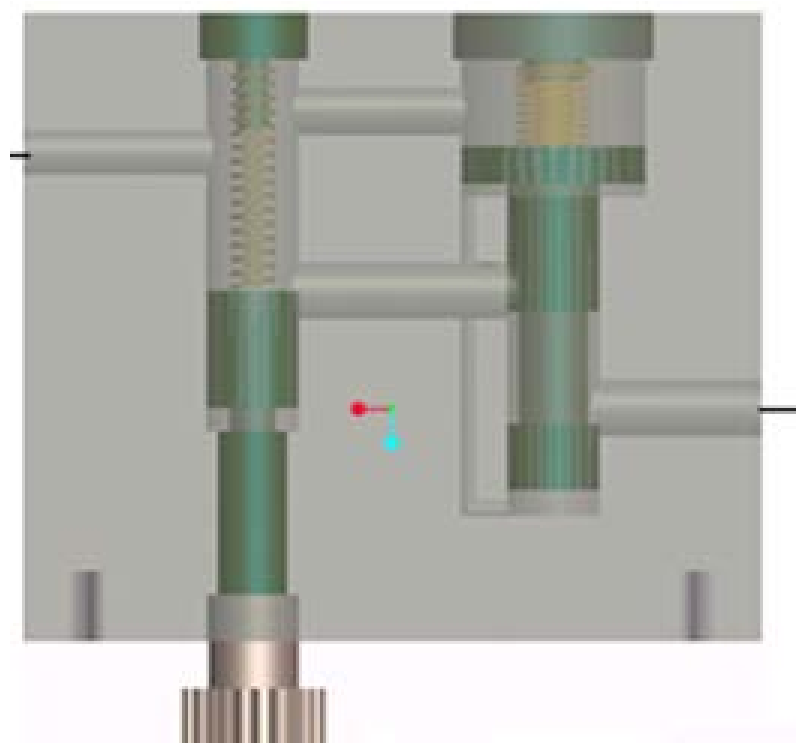

Figure 1. Speed control valve.

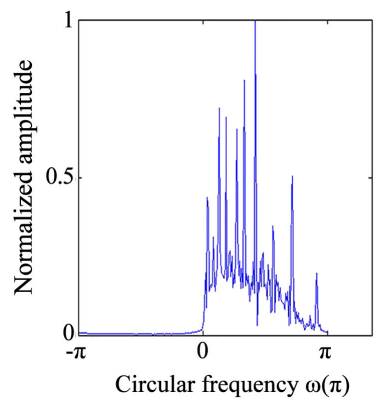

(a)

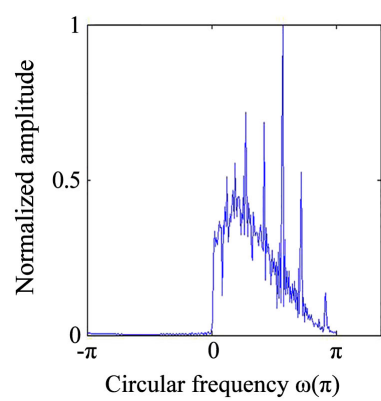

(b)

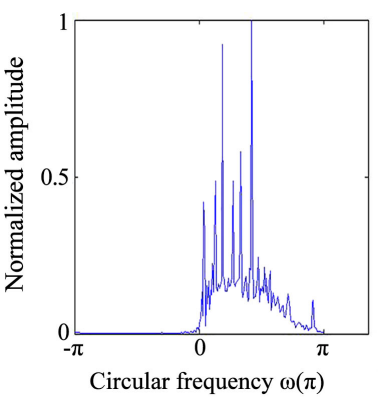

(c)

Figure 2. $1 \frac{1}{2}$ dimension spectrum of primary datum in definition 2. (a) Normal state; (b) Fault 1 state ( $3 \mathrm{MPa})$; (c) Fault 1 state $(5 \mathrm{MPa})$. 


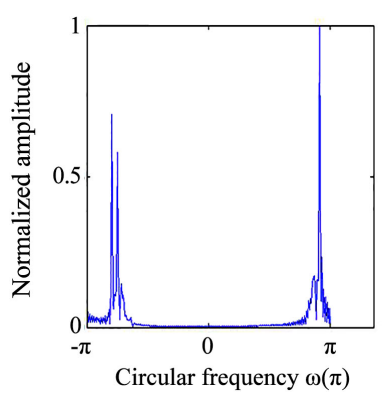

(a)

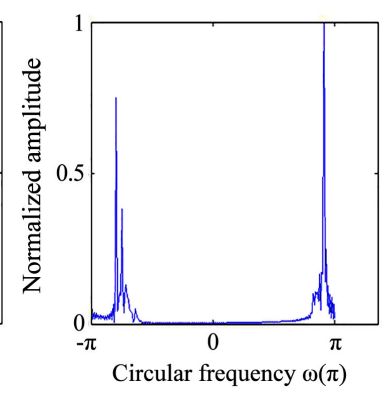

(b)

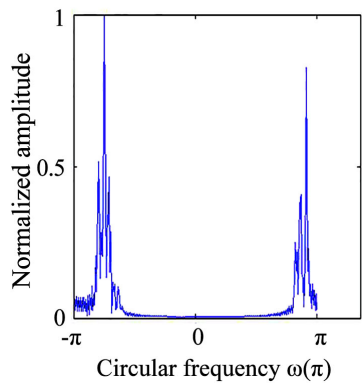

(c)

Figure 3. $1 \frac{1}{2}$ dimension spectrum of datum differeniated in definition 2. (a) Normal state; (b) Fault 1 state (3 MPa); (c) Fault 1 state ( $5 \mathrm{MPa})$.

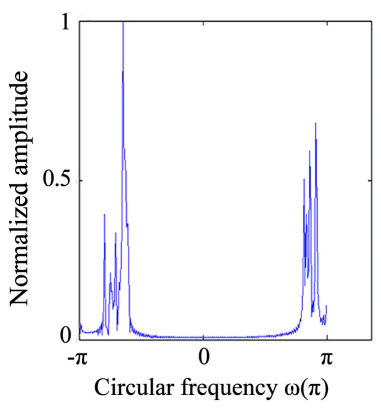

(a)

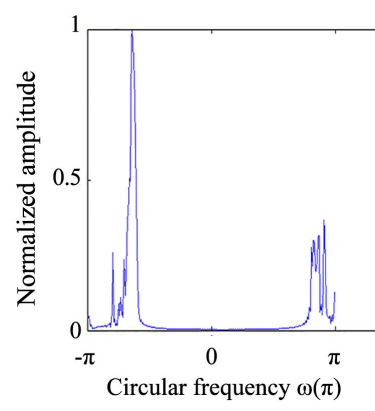

(b)

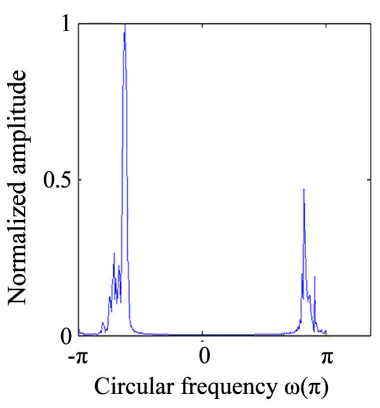

(c)

Figure 4. $1 \frac{1}{2}$ dimension spectrum of datum after differeniated 4 times in definition 2.

(a) Normal state; (b) Fault 1 state (3 MPa); (c) Fault 1 state (5 MPa).

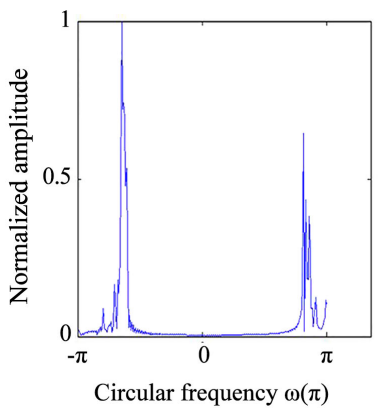

(a)

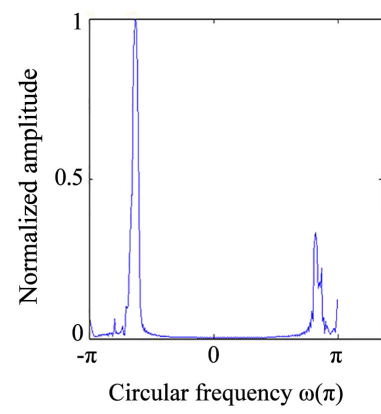

(b)

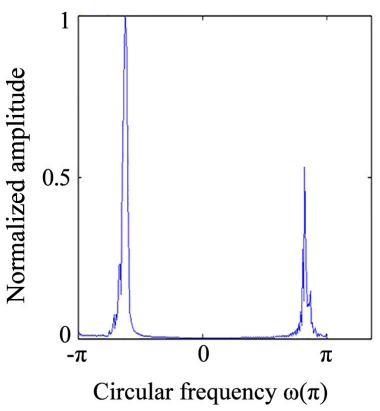

(c)

Figure 5. $1 \frac{1}{2}$ dimension spectrum of datum after differeniated 8 times in definition 2 . (a) Normal state; (b) Fault 1 state (3 MPa); (c) Fault 1 state (5 MPa).

Table 4. Capacity value of $1 \frac{1}{2}$ dimension in both normal state and fault 1 state after differentiated 8 times.

\begin{tabular}{ccccccccc}
\hline $\begin{array}{c}\text { Normal } \\
\text { state }\end{array}$ & 1.1910 & 1.1747 & 1.1796 & 1.1716 & 1.2193 & 1.1820 & 1.1178 & $\ldots$ \\
\hline $\begin{array}{c}\text { Fault } 1 \\
\text { state }\end{array}$ & 1.1513 & 1.2631 & 1.1052 & 1.1516 & 1.1470 & 1.1299 & 1.1636 & $\ldots$ \\
\hline
\end{tabular}




\section{The Experimental Results}

In order to effectively distinguish faults, fractal theory is used as a tool to calculate the capacity dimensions of complex signals' $1 \frac{1}{2}$ dimension spectrum, and all the datum which include above three different definition forms both in normal and fault state are calculated, the complex signals are obtained from original signals through Hilbert transform [12].

1): In definition two, the capacity dimensions of dotum's $1 \frac{1}{2}$ dimension spectrum (a total of 21 data, the 10 groups of normal state, 11 groups of fault one) are calculated, as shown in Table 4 . In order to effectively observe the results, they are drawn with Excel Data Point-fold Line Chart in Figure 6. The X-axis of Figure 6 represents the number of data, and the $\mathrm{Y}$-axis represents the capacity dimension calculated, dimensionless. As can be seen from Figure 6, the capacity dimensions of signals in normal state and fault state are difficult to distinguish in the whole. Then so do the definition one and definition three, the results are almost the same as Figure 6. Furthermore, the same steps are performed to real signals (without Hilbert transform). The result is still the same. All the above experimental results prove that, only depending on traditional spectrum analysis, some faults are difficult to distinguish. Then the differential method is adopted, after repeating the above steps, the results are shown in Figure 7. As shown, the numerical values of capacity dimension in normal state and fault state are still difficult to separate in the whole. Continue to increase the differential times, Figure 8 shows the results of 4 times. As can be seen in Figure 8, the discernibility degree of capacity dimensions gets better. In Figure 8, the fourth data of normal state points is designated as threshold, that is to say, if capacity dimension is greater than or equal to 1.1747 , this group of data is judged as normal state, else judged as fault one state, According to this approach, the number misjudged in normal state is 1 , and in fault one state is 5 , the correct rate in total is $70 \%$. When the differential times reach 8 , the results are shown in Figure 9. The same method is adopted as Figure 8 , the tenth data point of normal state is chosen as the threshold value, if capacity dimension is greater than or equal to 1.1558 , this group of data is judged as normal state, else judged as fault one state, the number misjudged in normal state is 1 , and in fault one state is 2 , the correct rate in total is $85 \%$. If continuing to increase the differential times, its correct rate does not rise, or even declined. In the whole, diagnostic correct rate firstly increases with the increase of differential times, reach a maximum, then even drops.

2): The same experiments are done in definition one, and when the differential times is 5 , the diagnostic correct rate reaches the maximum value of about $70 \%$. The obtained results of definition three are better than that of definition one, but worse than that of definition two.

\section{Analysis of Experimental Results}

According to Literature (7) and (8), $1 \frac{1}{2}$ dimensional spectrum can reflect well 
the signal frequency coupling features, and capacity dimension is used to calculate the similar ratio of complex graphics. In this paper, normal data or fault data is considered as one group respectively, capacity dimension of $1 \frac{1}{2}$ dimensional spectrum which contains the coupling properties is used to judge the differences in self similarity between the above two groups of datum. For fault one state, the experimental results show that, according to definition two mode, the best effect is reached, but in definition one mode is not so. As can be seen in Tables 1-3, in definition one mode, both three order cumulant and its diagonal slice are 0 , but in definition two, diagonal slice of cumulant is $2 A_{1} A_{2} A_{3} \omega_{1} \omega_{2} \omega_{3}\left[\exp j\left(\omega_{3} \tau+\pi / 2\right)\right]$. And with the increase of differential times, the amplitude becomes $A_{1} A_{2} A_{3} \omega_{1}^{n} \omega_{2}^{n} \omega_{3}^{n}$. Because diagnostic results get best after differentiated eight times, so it can be deduced that the frequency factors differented in amplitude do play a role in fault diagnosis. Fathomer, it can also be inferred that the frequencies difference must exist between normal state and fault one state, so with the increase of differential times, the differences become more obvious. The same experiments are carried out on other types of fault, and most results improve with the increase of differential times in a certain degree, but for a few of the above faults, the correct rates only improve little, even reduce. Considering the above experimental results, it can be extrapolated that, because of the complexity of mechanical vibration signals, only one coupling theory cannot fully explain their characteristics. Even so, the differential method in this paper succeeds in a certain degree. Because differential times are not limited, the methods provide an infinite choice of fault diagnosis. In Table 5, the highest correct rates comparison of three methods is listed.

\section{Conclusion}

The different definition forms of complex three order cumulants must contain

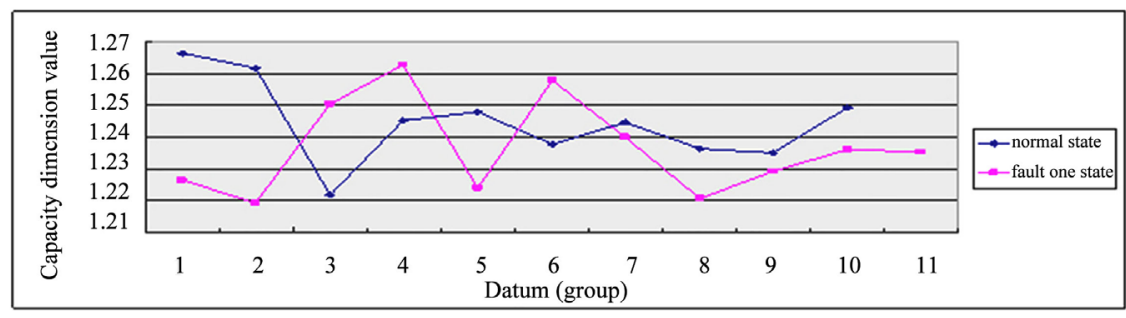

Figure 6. Simulation result of primary datum both normal state and fault 1 state in definition 2.

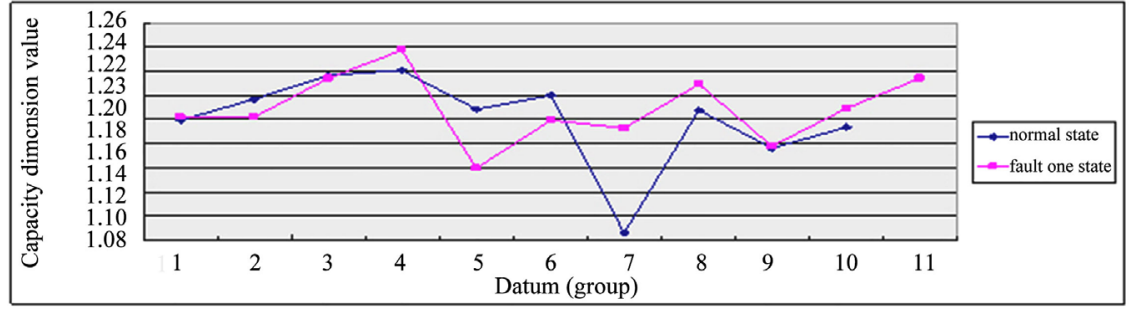

Figure 7. Simulation result of datum differentiated both normal state and fault 1 state in definition 2. 


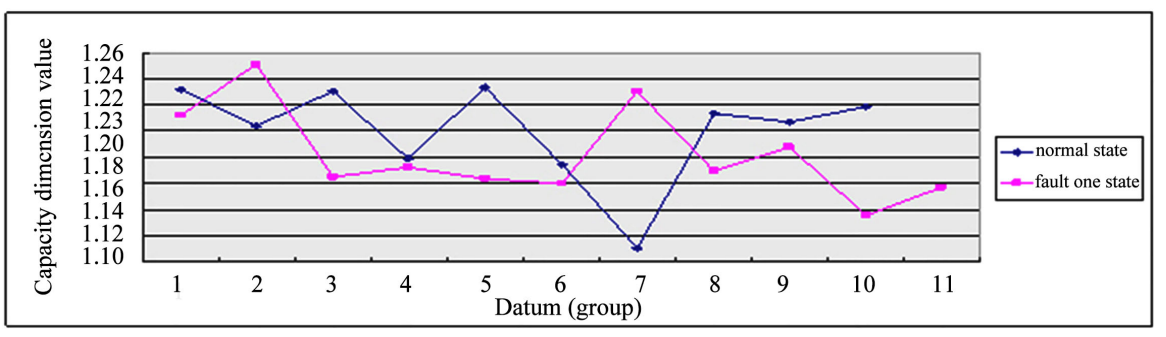

Figure 8. Simulation result of datum differentiated 4 times both normal state and fault 1 state in definition 2.

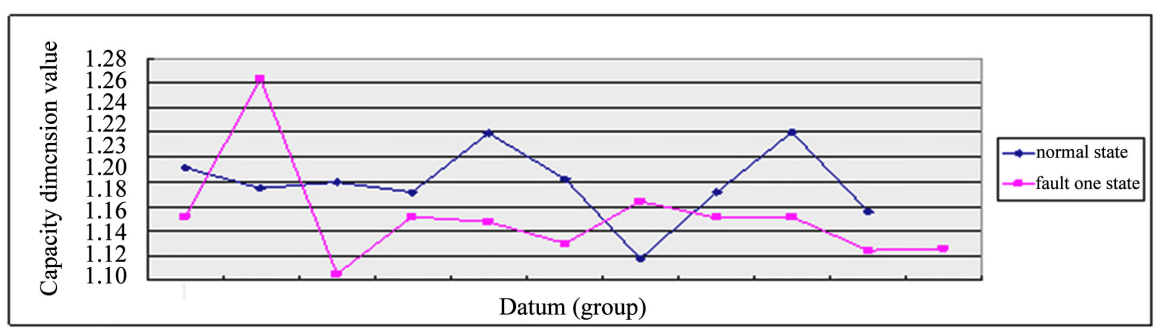

Figure 9. Simulation result of datum differentiated 8 times both normal state and fault 1 state in definition 2.

Table 5. The highest correct rates comparison of three methods.

\begin{tabular}{cccc}
\hline Method & Method in literature 10 & Method in literature 13 & Method in this paper \\
\hline highest correct rate & $85 \%$ & $80 \%$ & $95 \%$ \\
\hline
\end{tabular}

different coupling information, which is bound to react to the $1 \frac{1}{2}$ dimension spectrum derived from it. In the paper, differential operation is first performed on complex coupling signals, then the amplitude of three order cumulants derived from these differentiated signals includes the frequency components in the primary coupling signals, and these components are enlarged with the increase of differential times, afterwards, $1 \frac{1}{2}$ spectrums are obtained. Fault diagnosis based on computing capacity dimension of $1 \frac{1}{2}$ spectrum is performed, the results show that the frequency components included in three order cumulants after differentiated help to improve fault diagnostic correct rate.

\section{Acknowledgements}

This paper is supported by National Natural Science Foundation of China (61562063), Key Science and Technology Project of Jiangxi Provincial Education Department (GJJ161234).

\section{References}

[1] Feng, Y.X., Zhang, W.P., Yang, T., et al. (2015) Wind Turbine Planetary Gearbox Fault Analysis Based on Bispectrum. Advanced Materials Research, 1070-1072, 
1861-1868.

[2] Zhang, R.G. and Tan, Y.H. (2014) Intelligent Fault Diagnosis of Rolling Element Bearings Based on Bispectrum Principal Components Analysis. Zhendong Gongcheng Xuebaol Journal of Vibration Engineering, 27, 763-769.

[3] Wang, H., Deng, G., Li, Q., et al. (2016) Research on Bispectrum Analysis of Secondary Feature for Vehicle Exterior Noise Based on Nonnegative Tucker3 Decomposition. Eksploatacja i Niezawodnosc (Maintenance and Reliability), 18, 291-298. https://doi.org/10.17531/ein.2016.2.18

[4] Kesler, S.B. (1986) Modern Spectrum Analysis. IEEE Press, Piscataway, New Jersey.

[5] Marple, S.L. (1987) Digital Spectral Analysis with Applications. Prentice-Hall, Upper Saddle River, New Jersey.

[6] Raughveer, M.R. (1990) Time-Domain Approaches to Quadratic Phase Coupling Estimation. IEEE Transactions on Automatic Control, 35, 48-56.

https://doi.org/10.1109/9.45142

[7] Zhang, Y. and Wang, S.X. (1998) The Slice Spectra Approach to Nonlinear Phase Coupling Analysis. Acta Electronica Sinica, 26, 104-109.

[8] Zhang, Y., Wang, S.X. and Li, S.H. (1996) $1 \frac{1}{2}$-Dimension Spectrum Approach to Quadratic Phase Coupling Estimation. Acta Electronica Sinica, 64, 109-112.

[9] Wu, W.B., Yang, S.Q., Zhou, T.G. (2013) Application of Complex Three-Order Cumulants to Fault Diagnosis of Hydraulic Valve. Journal of Tianjin University (Science and Technology), 46, 590-595.

[10] Wu, W.B., Huang, Y.J. and Chen, W.X. (2012) High Order Spectrum's Coupling Performance Based on Mechanical Fault Diagnosis. Journal of Vibration, Measurement \& Diagnosis, 32, 130-134.

[11] Hinich, M.J. (1990) Higher Order Cumulants and Cumulant Spectra. 5th ASSP Workshop on Spectrum Estimation and Modeling, Rochester, NY, 10-12 October 1990, 391-402. https://doi.org/10.1109/SPECT.1990.205534

[12] Wu, W.B., Huang, Y.J. and Chen, W.X. (2013) The Impact of Complex Cumulants' Conpling Properties on the Results of Fault Diagnosis. Machine Design and Research, 29, 14-17. 